\title{
Nocardiosi: a proposito di tre casi
}

\section{Pietro Casella', Romualdo Grande', Maria Cristina Straface ', Massimo Pederzoli', Francesco Pitingolo ${ }^{3}$, Claudio Farina ${ }^{4}$}

'SMeL A.O. “Ospedale Civile di Vimercate”, Presidio di Vimercate

${ }^{2}$ Divisione di Neurologia, "Ospedale Civile di Vimercate", Presidio di Vimercate

${ }^{3}$ Divisione di Nefrologia e Dialisi, “Ospedale Civile di Vimercate”, Presidio di Vimercate

${ }^{4}$ U.O. Microbiologia, A.O. Ospedale S. Carlo Borromeo (Milano)

Key words: Nocardia

\section{Nocardiosis in Milan area (200I-2003): about three cases}

\section{SUMMARY}

We describe three case reports of nocardiosis occurred at the AO "Ospedale Civile di Vimercate", Vimercate (Milano) from 2001 to 2003.

The patients discussed are paradigmatic of some predisposing conditions. Case I was a kidney transplanted patient, and immunosuppression is a well-known jatrogenous underlying condition. Case 2 is paradigmatic of a systemic infection presented with predominant symptoms of CNS involvement: diabetes was a unique debilitating factor, and his activity as smith could represent a professional risk factor, because of the increased possibility to inhale dust. Case 3 represent an absolutely uncommon way of contamination in an immunocompetent woman receiving at home an i.m. therapy.

In all cases chemosensitivity testing showed sensitivity to imipenem, amikacin, cotrimoxazole. Antibiotic therapy was started with a combination of intravenous Imipenem plus Amikacin. The treatment was prolonged for two months and then was prescribed cotrimoxazole for eight months.

\section{INTRODUZIONE}

Al genere Nocardia appartengono circa 30 specie (2). Actinomiceti aerobi, sono ubiquitari nell'ambiente, in particolare giocano un ruolo fondamentale nella decomposizione della materia organica vegetale (2). Le infezioni umane da Nocardia sono piuttosto rare e colpiscono prevalentemente soggetti immunocompromessi (2).

La modalità di acquisizione dell'infezione più comune è quella inalatoria, e più raramente è quella traumatica, che comporta l'impianto diretto sulla cute (2).

La maggior parte dei casi di infezione invasiva nei paesi a clima temperato (circa $50 \%$ ) è causata dai batteri del complesso Nocardia asteroides (5). Le manifestazioni cliniche, la severità e la prognosi della malattia nei soggetti colpiti è variabile. La via di infezione e lo status immunologico del soggetto giocano un ruolo rilevante. In particolare, fattori predisponenti sono le terapie immunosoppressive, i trapianti, l'AIDS, le malattie broncopolmonari croniche ed il diabete.

\section{CASI CLINICI}

1. B.C., 52 anni, donna, normoergica. Il giorno 22/09/2003 si presentava al Pronto Soccorso dove le veniva drenato un ascesso al gluteo destro, insorto nella sede di una precedente iniezione intramuscolare effettuata a domicilio pochi giorni prima.
Nonostante la terapia antibiotica empirica con amoxicillina e ceftriaxone, la paziente continuava a presentare febbre intermittente. Il giorno 4/11/2003 venne nuovamente drenato l'ascesso ed avviate le indagini microbiologiche del materiale purulento. Gli esami ematochimici eseguiti evidenziavano leucocitosi $18400 \mathrm{WBC}$, con alterazione degli indici infiammatori (Proteina C Reattiva $3.9 \mathrm{mg} / \mathrm{dl}$ ). L'esame colturale del pus consentiva l'isolamento di Nocardia asteroides. Venivano eseguite TC torace e dell'encefalo, un'ecografia addominale ed un radiogramma del femore sinistro, tutti con esito negativo. Si iniziò terapia con sulfametossazolotrimethoprim 1 compressa x 2/die. Dal giorno $11 / 12$ la paziente era apiretica e riferiva completa cicatrizzazione della ferita cutanea. La paziente proseguiva la terapia per 3 mesi con piena risoluzione del quadro clinico.

2. I.F., 40 anni, donna, sottoposta ad intervento di trapianto renale due anni prima, e da allora in trattamento immunosoppressivo con tacrolimus, azatioprina e metilprednisolone. Circa un mese prima del ricovero la paziente riferiva, in assenza di traumi apparenti, la comparsa di un dolore acuto alla coscia destra con riscontro ecografico di distrazione del muscolo vasto mediale con ematoma. In giugno 2001 venne ricoverata per il persistere di sintomatologia algica e per l'estensione dell'e- 
matoma con la contemporanea comparsa di segni di flogosi: il drenaggio dell'ematoma consentiva l'evacuazione di materiale purulento in un quadro di iperpiressia $\left(38^{\circ} \mathrm{C}\right)$. Gli esami ematochimici evidenziavano leucocitosi neutrofila (17500 WBC) ed incremento degli indici di flogosi (VES 123, Proteina C Reattiva 15.0). Dal contenuto asessuale venne isolata Nocardia asteroides.

Venne instaurata terapia antibiotica sistemica con ceftriaxone e sulfametossazolo-trimethoprim, che comportava la pronta defervescenza e la normalizzazione degli indici di flogosi con riduzione dell'edema. Un'ecografia di controllo documentò completa risoluzione del processo infettivo. Venivano eseguite TC torace, encefalo con ecografia addominale risultate negative. La paziente proseguiva terapia con sulfametossazolo e trimethoprim per 6 mesi con piena risoluzione del quadro clinico.

3. A.A., 61 anni, uomo, diabetico e iperteso, di professione fabbro. Giunge all'osservazione presso la Divisione di Neurologia in data 13 aprile 2002 per comparsa subacuta di ipostenia dell'arto inferiore destro. Nel passato prossimo era segnalata una broncopolmonite. La TC dell'encefalo eseguita in regime di urgenza dimostrò la presenza di due lesioni di incerta natura in sede rispettivamente temporale destra e parietale posteriore sinistra. I primi esami di laboratorio posero in particolare evidenza una notevole leucocitosi neutrofila (24000 WBC). Il paziente era vigile, cosciente e presentava una lieve emisindrome motoria.

Durante il ricovero presentò ipertermia febbrile di tipo settico con elevazioni serotine fino a $39^{\circ} \mathrm{C}$. Il giorno 23 aprile vennero eseguite emocolture. La Tomografia-RM dell'encefalo confermò i reperti mostrati dall'esame TC, ponendo il sospetto di ascessi cerebrali di origine infettiva.

Pochi giorni dopo si svilupparono due tumefazioni cutanee voluminose e dolenti in sede, rispettivamente, scapolare destra ed addominale sinistra. Il giorno 26 aprile venne eseguita l'agobiopsia "ecoguidata" allo scopo di chiarirne la natura asessuale. Dall'emocoltura e dalla coltura del materiale purulento venne isolata Nocardia asteroides.

Venne instaurata terapia chemiantibiotica con imipenem cilastatina $500 \mathrm{mg}$ x 3/die per 6 settimane. Il trattamento determinò un rapido miglioramento delle condizioni generali, apiressia, riduzione delle tumefazioni visibili e del dolore locale, riduzione progressiva della leucocitosi fino alla normalità con miglioramento del quadro neurologico e riduzione progressiva degli ascessi multipli senza che fossero proposte opzioni di trattamento invasivo neurochirurgico, mentre l'ascesso muscolare scapolare venne drenato. Il 21 maggio il paziente fu dimesso, con terapia domiciliare con sulfametossa- zolo trimethoprim che continuò per 8 mesi fino alla completa risoluzione del quadro clinico.

\section{MATERIALI E METODI}

I campioni biologici sono stati seminati su Schaedler agar, CNA Agar e kanamicina/vancomicina Agar, incubate in aerobiosi ed in anaerobiosi, in aggiunta ad una piastra di Mc Conkey agar in aerobiosi, una piastra di agar sangue Columbia in anaerobiosi e di thioglicollato medium in anaerobiosi per $28 / 72$ ore a $35^{\circ} \mathrm{C}$.

Le emocolture sono state eseguite con il sistema BactT Alert (bioMérieux).

L'identificazione degli isolati è stata condotta presso il Centro Coordinatore per l'Italia del Programma di Sorveglianza epidemiologica proposta dall'European Confederation of Medical Mycology: "Epidemiological Survey on Nocardiosis and other aerobic actinomycetes infections in Europe" (Claudio Farina).

I test di sensibilità sono stati condotti con $\mathrm{E}$ test utilizzando Mueller Hinton agar più $5 \%$ di sangue di pecora, con incubazione a 48 ore in aria a $35^{\circ} \mathrm{C}$. Gli antibiotici saggiati sono stati i seguenti: eritromicina, tetraciclina, amoxicillina/acido clavulanico, cefotaxime, imipenem, amikacina, ciprofloxacina e sulfametossazolo trimethoprim (2).

Dall'esame diretto condotto sui materiali asessuali dei pazienti vennero evidenziati batteri Gram positivi filamentosi, ramificati, sottili e colorati irregolarmente.

Dopo 48 ore di incubazione in tutti i tre casi erano presenti colonie di aspetto puntiforme in Schaedler e CNA agar in aerobiosi. Nel paziente A.A. il ceppo venne isolato anche da emocoltura. Le colonie erano costituite da bacilli Gram positivi aerobi obbligati a crescita lenta che dopo diversi giorni assumevano l'aspetto di polvere di gesso le cui cellule presentavano morfologia microscopica descritta precedentemente.

I risultati dell'antibiogramma eseguiti con E test per i tre ceppi sono riportati in tabella 1

All'interno dell'alone di inibizione dell'imipenem erano presenti microcolonie (figura I). Se queste colonie costituite da cloni apparentemente resistenti venivano isolate e l'antibiogramma era ripetuto, questi cloni mostravano completa sensibilità.

\section{DISCUSSIONE}

Le Nocardiae si sviluppano sulla maggior parte dei terreni a libera crescita usati per l'isolamento di batteri, funghi e micobatteri e nei sistemi per emocolture: il loro isolamento potendo richiedere tuttavia diversi giorni, pare opportuno prolungare l'incubazione di materiali normalmente sterili come liquor o sangue per almeno dieci giorni. 
Tabella I. MIC ottenute per i tre ceppi di Nocardia isolati

\begin{tabular}{lllllllll}
\hline Paziente & eritrom. & tetrac. & amoxi/clav & cefotaxime & imipenem & amikacina & cotrimox & cipro. \\
\hline B.C. & 32 & 16 & 1 & 32 & $\begin{array}{l}2 \\
(32)^{*}\end{array}$ & 0.5 & 0.06 & 2 \\
\hline I.F. & 32 & 16 & 1 & 32 & $\begin{array}{l}2 \\
(32)\end{array}$ & 0.5 & 0.125 & 0.06 \\
\hline A.A. & 32 & 16 & 4 & 32 & 4 & 0.5 & 0.125 & 0.125 \\
\end{tabular}

* I risultati tra parentesi indicano la MIC letta considerando microcolonie puntiformi sviluppatesi allinterno dell'ellissometro più evidente.

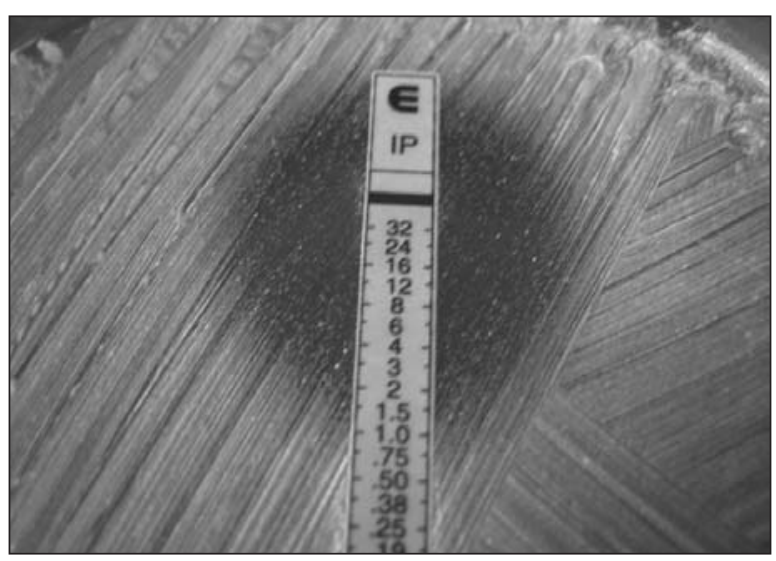

Figura I. E test con imipenem: microcolonie all'interno dell'ellisometro

Alcuni ceppi, inoltre, perdono vitalità a basse temperature: per tale motivo campioni biologici come i materiali respiratori (lavaggio broncoalveolare o espettorato) non andrebbero refrigerati, e seminati anche su terreni selettivi come Tayer Martin Agar o BCYE Agar per facilitarne la crescita. La lentezza di sviluppo di Nocardia e l'uso di terreni selettivi diversi da quelli descritti nei caso di materiali polimicrobici potrebbe spiegare in parte il loro raro riscontro.

L'esame microscopico diretto di un preparato colorato secondo Gram è di fondamentale importanza per confermare o indirizzare un sospetto clinico di infezione da Nocardia.

La comparsa di colonie visibili in aerobiosi dopo 23 giorni, di aspetto puntiforme che dopo 5-6 giorni appaiono con aspetto di polvere di gesso, per la presenza di micelio aereo, e di colore variabile dal bianco al rosa salmone con morfologia microscopica simile a quella eventualmente riscontrata nei preparati diretti è stata una caratteristica che ha consentito di identificare i ceppi in via preliminare come appartenenti al genere Nocardia.

La terapia delle nocardiosi deve tener conto della localizzazione dell'infezione, della sua severità, dello status immunologico del paziente e della potenziale tossicità del farmaco. I test in vitro rappresentano un'utile guida per una corretta gestione della terapia soprattutto per le infezioni gravi $(2,4)$. Non essendo stati stabiliti valori di break point è razionale condurre un test in diluizione $\mathrm{e}$ riferire solo la MIC, lasciando al clinico infettivo- logo il compito di stabilire la strategia ottimale di trattamento.

Nella nostra esperienza E test si è rivelato particolarmente maneggevole. Per le nocardiosi E test è stato segnalato come un'agevole alternativa all'esecuzione delle MIC classiche (4): la crescita di cloni apparentemente resistenti all'interno dell'ellissometro di inibizione di imipenem potrebbe indurre ad una scorretta interpretazione della MIC, con il rischio di sovrastimare la resistenza. La terapia di scelta per infezioni da Nocardia sia localizzate che disseminate è rappresentata da cotrimossazolo (4). Altri antibiotici come imipenem ed amikacina, da soli o in associazione, anche a sulfametossazolo trimethoprim sono stati usati con successo nelle infezioni da Nocardia (4). Nei due casi localizzati da noi descritti, la risoluzione con cotrimossazolo somministrato per sei mesi era attesa. Il dato più significativo resta la completa risoluzione dell'infezione sistemica nel paziente con ascessi cerebrali non aggredibili chirurgicamente. In questi la mortalità può raggiungere il $41 \%$.

\section{BIBLIOGRAFIA}

1. Acar T, Arshad M. Nocardia asteroids cerebral abscess in a renal transplant recipient: short report. Acta Chir Belg Dec ;102 (6): 470-1.

2. Brown JM, Mc Neil M. Nocardia, Rhodococcus, Gordonia, Actinomadura, Streptomyces, and Other Aerobic Actinomycetes. Murray PR Ed. In Manual of Clinical Microbiology $8^{\text {th }}$. ASM Press. Whashington, D.C.

3. Saubolle MA, Sussland D. Nocardiosis: review of clinical and laboratory experience. J Clin Microbiol 2003; 41(10): 4497-501.

4. Sorrell T C, Iredell J R, Mitchell D H. Nocardia species. In Principles and Practice of Infectious Diseases. Mandell GL, Bennette JE, Dolin R. fifth Edition, 2000; V2: 2637-2645.

5. http://www.cdc.gov/ncidod/dbmd/diseaseinfo/nocardiosis t.htm

\section{Pietro Casella}

SmeL, A.O. "Ospedale Civile di Vimercate" Presidio di Vimercate

Via C. Battisti 23 - 20059 Vimercate (MI)

Tel: 039 6654654; Fax: 0396654772

E-mail: casella.pietro@aovimercate.org 